\title{
Comparative adherence to oxybutynin or tolterodine among older patients
}

\author{
Tara Gomes • David Juurlink • Muhammad Mamdani
}

Received: 12 January 2012 / Accepted: 13 January 2012 / Published online: 8 February 2012

(C) Springer-Verlag 2012

Letter to the Editor: Response

In their letter, Thompson and Dahlqvist suggest that the findings of our paper describing the comparative adherence to anticholinergic drugs could be improved by the addition of details regarding the formulations used by patients in our cohort. They note that incidence of dry mouth-a commonly cited reason for drug discontinuation - is most often observed with immediate-release formulations. In Ontario, only immediate-release (IR) formulations of oxybutynin are funded through the public drug plan; consequently, $99.6 \%$

T. Gomes $(\bowtie) \cdot$ D. Juurlink $\cdot$ M. Mamdani

Institute for Clinical Evaluative Sciences,

Toronto, Ontario M4N 3M5, Canada

e-mail: tara.gomes@ices.on.ca

T. Gomes $\cdot$ M. Mamdani

Leslie Dan Faculty of Pharmacy, University of Toronto,

Toronto, Ontario, Canada

D. Juurlink

The Sunnybrook Research Institute, St. Michael's Hospital,

Toronto, Ontario, Canada

D. Juurlink

Department of Pediatrics, University of Toronto,

Toronto, Ontario, Canada

D. Juurlink $\cdot$ M. Mamdani

Departments of Medicine and Health Policy,

Management and Evaluation, University of Toronto,

Toronto, Ontario, Canada

M. Mamdani

Department of Medicine, St. Michael's Hospital,

Toronto, Ontario, Canada

M. Mamdani

Keenan Research Centre of the Li Ka Shing Knowledge Institute,

St. Michael's Hospital,

Toronto, Ontario, Canada of patients initiating oxybutynin in our study were treated with an IR tablet formulation. However, both IR and extended-release (ER) formulations of tolterodine are publically funded in Ontario, and therefore only $58.9 \%$ of patients newly treated with tolterodine commenced treatment with an IR formulation. In response to Thompson's and Dahlqvist's comments, we replicated our previous analysis, comparing persistence among 31,990 patients initiating IR oxybutynin with that of 14,637 patients initiating IR tolterodine derived from our original cohort. Similar to our earlier study, we found a significant difference in persistence rates between people treated with IR oxybutynin and IR tolterodine $(p<0.0001$; Fig. 1). After 6 months of followup, only $30.6 \%$ of new IR oxybutynin users persisted with therapy compared to $45.9 \%$ of new IR tolterodine users.

These updated findings suggest that differences in adherence to oxybutynin and tolterodine persist among a subgroup of patients initiated on IR formulations.

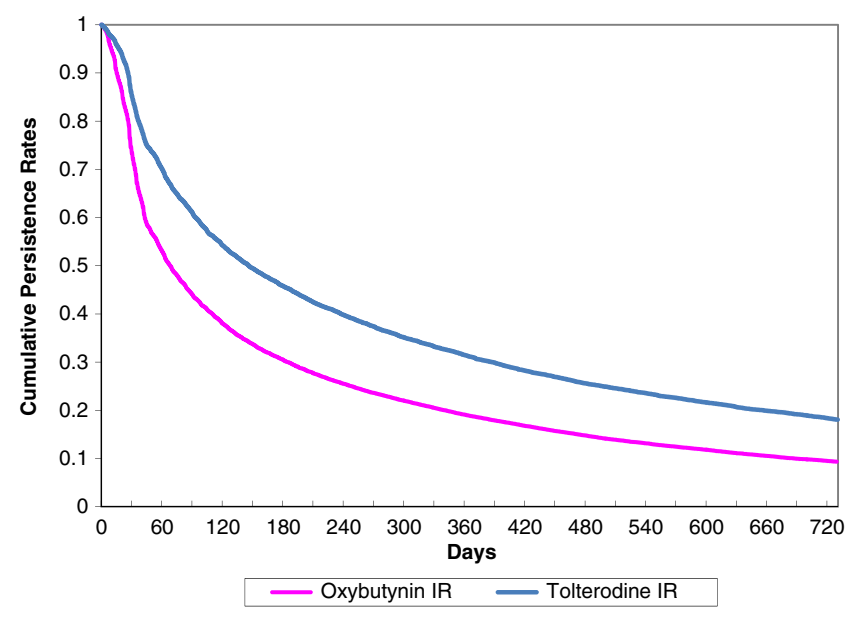

Fig. 1 Cumulative rate of persistence among elderly patients newly prescribed immediate-release oxybutynin tablets and immediaterelease tolterodine capsules between January 2000 and December 2007 